\title{
Editorial: Rock Physics and Geofluid Detection
}

\author{
Jing Ba ${ }^{1,2 *}$, Zhijian Fang ${ }^{2}$, José M. Carcione ${ }^{2,3}$, Li-Yun Fu ${ }^{4}$ and Qiang Guo ${ }^{2,5}$ \\ ${ }^{1}$ State Key Laboratory of Shale Oil and Gas Enrichment Mechanisms and Effective Development, Beijing, China, ${ }^{2}$ School of Earth \\ Sciences and Engineering, Hohai University, Nanjing, China, ${ }^{3}$ National Institute of Oceanography and Applied Geophysics (OGS), \\ Trieste, Italy, ${ }^{4}$ School of Geosciences, China University of Petroleum (East China), Qingdao, China, ${ }^{5}$ College of Information \\ Engineering, China Jiliang University, Hangzhou, China
}

Keywords: rock physics, geofluid detection, theories, experiments, geophysical exploration

Editorial on the Research Topic

\section{Rock Physics and Geofluid Detection}

Rock physics is the discipline that allows the characterization of the properties of rocks mainly based on theoretical and experimental non-destructive methods. The effect of pore fluids is of interest due to its applicability to the hydrocarbon industry. The methods involve wave propagation and low-frequency tests on field samples and seismic and electromagnetic experiments and models under varying conditions of saturation and pore pressure. This requires the establishment of constitutive relations with particular attention to the interaction between the mineral components and the saturating fluids. The relations can be of elastic, electromagnetic, or diffusive (flow) nature. Geophysical methods aim to obtain information about fluid type, porosity, permeability, mineralogy, and the presence of cracks and fractures. In this sense, experiments, mathematical theories, and numerical algorithms are essential for a quantitative characterization of subsurface geological structures, in-situ conditions, and for the detection of geofluids. Rock physics is applied in different fields, such as geology, geophysics, petrophysics, mining and resource exploration, hydrology and natural hazards.

This research topic, "Rock Physics and Geofluid Detection," contains high-quality original research contributions regarding novel methodologies and applications to characterize the physical behavior of rocks aiming at geofluid detection. The purpose is to solve the pivotal problems related to theories/ methods and prospective technologies from different areas which can be helpful for improving the developments of accurate exploration methods. The twenty-five papers collected mainly cover the following four subtopics.

\section{NOVEL ROCK-PHYSICS EXPERIMENTS, THEORETICAL APPROACHES, AND NUMERICAL MODELING}

Received: 02 August 2021

Accepted: 24 August 2021

Published: 10 September 2021

Citation:

Ba J, Fang Z, Carcione JM, Fu L-Y and Guo Q (2021) Editorial: Rock Physics and Geofluid Detection.

Front. Earth Sci. 9:752172. do: 10.3389/feart.2021.752172

The twelve papers collected in this subtopic mainly include laboratory measurements of rock properties, wave propagation theories, and numerical modeling.

Deng et al. conducted a detailed petrological and geochemical analysis, and microstructure observation of the shale samples of the Wufeng-Longmaxi Formation in southeastern Sichuan Basin, and tested their acoustic and geochemical behaviors. This integrated study provides important insight into the reservoir parameters that control the dynamic and static elastic behaviors of shale.

By studying the propagation of waves in porous media with a specific structure in saturating immiscible fluids, Xiong et al. established a wave equation system with a relatively simple structure. 
This study improves the stability of the Tuncay-Corapcioglu model based on the volume average method.

Fu et al. studied the effect of the crack surface roughness on the elastic stiffness of dry and saturated rocks. Rough surfaces cause stress concentration around the cracks, reducing the elastic modulus of the rock.

Ikeda et al. proposed a method to predict the spatial distribution of the elastic properties in multi-mineral rocks, based on digital rock physics. It uses the homogenization theory and overcomes the monomineralic limitation. The method is applied to a carbonate sample, composed of dolomite and calcite.

Tisato et al. measured seismic wave attenuation and dynamic Young's modulus of dry and partially-saturated Berea sandstone within the range of $1-100 \mathrm{~Hz}$ and $0-20 \mathrm{MPa}$ confining pressure. The data was compared with those predicted by phenomenological models of ultrasonic elastic properties and wave-induced-fluid flow. The analysis shows that the attenuation is strongly dependent on pressure. This result can help interpreting near-surface geophysical data and improve subsurface imaging.

In order to study the effect of nanopore distribution on the pressure sensitivity of P-wave velocity in dry rocks, Raj et al. used a set of 23 samples of a vertical core collected from the Mississippian-age mixed carbonate-siliciclastic Meramec formation. The analysis shows that the velocitypressure relation depends on the distribution of pore shape and size.

Cao et al. considered the elastic interaction between cracks to investigate the effective elasticities in complex fractured media. They compared the analytical solution without stress interaction and the numerical result with interaction. The results show that regardless of the spatial distribution of the cracks, their size determines the dominant type of interaction, significantly affecting the effective properties.

$\mathrm{Ma}$ et al. studied the fluid sensitivity to petrophysical properties based on experimental data by using fluid indicators and crossplots, which can discriminate well between gas and liquids (i.e., water and oil). Siltstone and dolomite can be identified according to the distribution of the data (the area specifically).

Based on modeled low-frequency longitudinal forced oscillations, Mikhaltsevitch et al. studied the effect of the strain gauge location on the measurements, the creep effect related to the rock mineralogy, and the dispersion/attenuation in a liquid-saturated rock sample caused by the existence of an external pore fluid volume (dead volume). The results are independent of the location of the strain gauges, the lowfrequency moduli are noticeably reduced with time, and the relaxation frequency is independent of the dead volume.

Spikes and Sen analyzed the inherent dependence and correlation among the input parameters of a petrophysical model by using a Bayesian framework. This method takes into account the fluid type and saturation, mineralogy, pressure, and critical porosity. When applied to well data, the results show that there is a similar correlation between pressure and critical porosity in dry and clean sands.
Charoensawan et al. modeled the P-wave velocity in Alpine fault rocks with and without microfractures. The results reveal the influence of the crack orientation and shape. Anisotropy, clearly observed in the shear zone of the mylonite Alpine fault, is caused by different minerals and structural features and becomes more complex due to the presence of cracks.

Cheng et al. performed ultrasonic measurements on 15 tight gas sandstone samples of the Xujiahe formation in western Sichuan under different confining pressures. By analyzing log and seismic data, a 3-D rock-physics template was established. The estimated stiff porosity and crack porosity are consistent with data from two wells.

\section{HIGH-PRECISION INVERSION OF STRATA PROPERTIES FROM GEOPHYSICAL DATA}

The three papers collected in this subtopic deal with stabilityenhanced, high-resolution and rock-physics-model-based linear and nonlinear inversion schemes applied to geophysical data.

Yu et al. proposed a model to describe the elastic properties of mud-rich source rocks, filled with kerogen at different maturity stages. The model provides a theoretical basis for seismic forward modeling and inversion and allows the characterization of this type of unconventional resource.

Jin et al. obtained the velocity dispersion and attenuation caused by fluid squirt flow in tight-gas sandstones with a complex pore space. Frequency-dependent AVO and inversion of a $\mathrm{P}$-wave dispersion attribute are studied as a function of gas saturation. Higher saturation implies lower P-wave velocity and stronger attenuation. The inversion of synthetic data shows that the attribute is an effective indicator of saturation.

Avseth and Lehocki proposed a new integrated workflow for generating AVO feasibility maps/cubes by using data from the Barents Sea. This method can quickly extrapolate away from the expected rock physics characteristics of well control along the selected horizon, which is constrained by seismic velocity information, geological input and rock physics properties. This workflow allows faster, seamless, and geologically consistent direct hydrocarbon identification to reduce prospecting risks in the areas with complex geological and tectonic impacts.

\section{GEOFLUID DETECTION WORKFLOWS AND APPLICATIONS WITH CASE STUDIES}

The four papers collected in this subtopic include new techniques, methods, and workflows for detecting geofluids and the verification by comparing theories and real data.

Vesnaver et al. developed a new method for imaging seismic attenuation in a seismic survey acquired at the Gulf of Trieste (Italy). The method combines the macroscopic model obtained by seismic tomography with the microscopic model obtained by seismic attributes of instantaneous frequency, leading to a highresolution image of $Q$.

Zhou et al. developed a rock-physics template based on a double double-porosity theory, which could effectively identify 
fluids in ultra-deep reservoirs. The template is applied to faultcontrolled karst reservoirs in the Tarim Basin (China), revealing ultra-deep oil and gas resources with diverse pore-crack systems, low permeability, and complex spatial distributions of oil and water.

Balcewicz et al. combined a high-resolution image from X-ray tomography, transmission optical microscope and scanning electron microscope with attributes such as geological diagenesis, mineral composition, microstructure and porosity to obtain the best spatial segmentation image of a Ruhr sandstone. The image histogram is compared with a numerical simulation, providing a workflow to describe the microstructural characteristics of the sandstone.

Alabbad et al. proposed a deterministic seismic interpretation method, based on rock physics to discriminate pore fluids and quantify the porosity and mineralogy from seismic impedance. The method was applied to carbonate and clastic oil fields. The pore fluid plays a role in the evolution of porosity.

\section{LABORATORY AND FIELD MODELS AND APPLICATIONS}

The six papers collected in this subtopic use rock physics by combining field and experimental data, to predict reservoir properties.

Dvorkin et al. analyzed wireline data from Wolfcamp and Woodford gas shales and showed that porosity and mineralogy control the elastic properties. A model is established based on digital images to estimate these properties from small irregular rock fragments.

Huang et al. proposed a model of seismic anisotropy to perform forward modeling. The model can be used to characterize the anisotropic properties of a Precambrian carbonate with fractured vuggy pores and caves.

$\mathrm{Xu}$ et al. combined discrete element models with discrete fracture networks. The method provides a simple approach with a high computation efficiency to achieve a realistic and detailed description of a fracture system.

Guo et al. established three models to describe the complex pore space of tight-gas sandstone reservoirs. One model assumes a single pore aspect ratio, another considers hard and soft pores, and third model is based on the mean and variance aspect ratios to represent a statistical distribution. The accuracy of the models is verified by numerical simulations.

Gas hydrate is a new energy resource with great potential, widely distributed in permafrost and seabed sediments. Rock physics is useful to study wave dissipation with increasing hydrate concentration at different frequencies. Two relevant papers are:
Wang et al. considered two different rock-physics models to analyze gas-hydrate bearing sediments based on the BiotRayleigh double-porosity theory. The theoretical results agree with the experimental data. In particular, at sonic frequencies one of the models predicts high attenuation due to wave-induced local fluid flow. Moreover, the analysis reveals that attenuation increases up to a given hydrate concentration and then decreases.

Guo et al. introduced a critical hydrate saturation indicator to describe the type of pore filling and the presence of grain cementation. This indicator is implemented in a petrophysical model to compute the P-wave velocity dispersion and attenuation caused by squirt flow. When the gas-hydrate saturation is less than the critical one, the velocity slightly increases with increasing saturation, while the velocity increases significantly in the presence of cementation.

In summary, the research topic covers a wide range of methodologies and approaches which provide fundamental insights into geofluid detection. These works are useful to show how to integrate different disciplines, which facilitate the improvement of accurate exploration methods based on rock physics.

\section{AUTHOR CONTRIBUTIONS}

All authors listed have made a substantial, direct, and intellectual contribution to the work and approved it for publication.

\section{ACKNOWLEDGMENTS}

We thank all authors for their valuable contributions to this research topic, the reviewers who shared their precious time to improve the manuscripts, the members of the Frontiers Team and Editor in Chief for their help at different stages of preparing this collection of papers.

Conflict of Interest: The authors declare that the research was conducted in the absence of any commercial or financial relationships that could be construed as a potential conflict of interest.

Publisher's Note: All claims expressed in this article are solely those of the authors and do not necessarily represent those of their affiliated organizations, or those of the publisher, the editors and the reviewers. Any product that may be evaluated in this article, or claim that may be made by its manufacturer, is not guaranteed or endorsed by the publisher.

Copyright (C) $2021 \mathrm{Ba}$, Fang, Carcione, Fu and Guo. This is an open-access article distributed under the terms of the Creative Commons Attribution License (CC BY). The use, distribution or reproduction in other forums is permitted, provided the original author(s) and the copyright owner(s) are credited and that the original publication in this journal is cited, in accordance with accepted academic practice. No use, distribution or reproduction is permitted which does not comply with these terms. 